\title{
Max Karl Ernst Ludwig Planck
}

Physicien théorique, professeur à la prestigieuse Université Friedrich-Wilhelms de Berlin, musicien doué, Max Planck est surtout connu pour avoir révolutionné la perception des lois de la physique et introduit la notion de quanta d'énergie. On lui doit notamment d'avoir établi la loi de répartition spectrale du rayonnement thermique du corps noir. La constante universelle $h$ porte son nom, et ses travaux ont servi de socle à l'explication de l'effet photoélectrique par Albert Einstein et à la description de l'atome par Niels Bohr.

$\mathrm{M}$ ax Planck, de son nom de baptême Marx, naît le 23 avril 1858 à Kiel, la capitale du Länder allemand SlesvigHolsace, à la frontière avec le Danemark, dans une famille d'universitaires. Son grand-père et son arrière-grand-père paternels enseignaient la théologie à Göttingen, et son père Johann Julius Wilhelm Planck est professeur de droit constitutionnel à l'Université de Kiel. Sa mère, Emma Patzig, issue d'une famille de pasteurs, est la seconde femme de Johann. À la naissance de Max, la famille compte cinq enfants (dont deux de la première union du père avec Mathilde Voigt) et ses parents sont déjà relativement âgés. II est élevé dans le respect des valeurs traditionnelles, et l'éducation scolaire revêt une importance particulière.

Max fréquente l'école élémentaire à Kiel jusqu'en 1867, I'année du déménagement des Planck pour Münich où son père est muté. C'est un changement d'univers : Kiel est une petite ville portuaire et provinciale; Münich est une capitale économique et culturelle, au centre d'une région lacustre et à proximité immédiate de massifs montagneux célèbres pour leurs circuits de randonnée. Le jeune Max s'y épanouit.

Passionné notamment par la musique, il montre un vrai talent au piano et à I'orgue. Il entre au Maximilian Gymnasium, où il se révèle un élève studieux et régulier, à l'esprit clair et logique. Son goût pour les sciences se manifeste un peu plus tard, et se développe sous la férule de son professeur Hermann Müller.

\section{Les études}

En 1874, il obtient son Abitur avec mention et, après une période $d$ 'indécision où il hésite pour une carrière musical et, il opte pour les sciences et intègre l'Univer- sité de Münich en octobre. Les premiers mois, Max Planck se cherche encore. II suit assidûment les cours de mathématiques, puis de physique. Séduit, il interroge son professeur Philipp von Jolly sur les perspectives d'une carrière de chercheur ; celui-ci lui décrit une science achevée, maîtrisée, au périmètre parfaitement délimité. Il est fidèle en cela à la perception générale des physiciens de la fin du 19 e siècle... Néanmoins, malgré ce tableau noir d'un horizon bouché, Planck persiste et poursuit son cursus.

Une maladie le cloue chez lui tout l'été 1875, il doit même interrompre ses études pendant plusieurs mois. Une fois rétabli, il décide de rejoindre l'Université de Berlin, où il suit les cours de Weierstrass [1815-1897], von Helmholtz [1821-1894] (un professeur brouillon, avec qui il se lie d'amitié) et Kirchhoff [1824-1887] (plus soigneux, mais monotone). Il entreprend d'étudier seul les travaux de Clausius sur la thermodynamique, et s'enthousiasme pour la beauté conceptuelle du second principe sur l'évolution de l'entropie.

Il boucle son cursus universitaire en 1878, et rentre à Münich pour préparer un doctorat. II soutient sa thèse sur le second principe de théorie mécanique de la chaleur en Juillet 1879, un sujet peu conventionnel mais qui convainc le jury et lui vaut la mention summa cum laude.

Un an plus tard, Planck passe son habilitation sur les états d'équilibre des corps isotropes à différentes températures. II devient ainsi privat-docent à l'Université de Münich. C'est un poste d'enseignant précaire et non rémunéré, qui l'oblige à vivre chez ses parents et retarde ses projets matrimoniaux. C'est cependant un passage obligé pour obtenir une chaire, et Planck y consacre cinq années.

En 1885, il décroche un poste de profes-

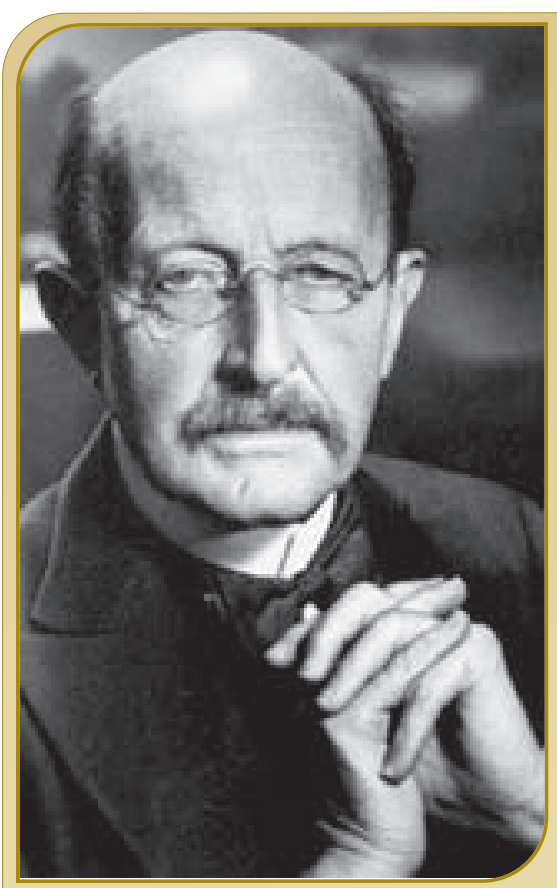

Principales dates

23 avril 1858

Naissance à Kiel (Allemagne)

1879 Doctorat sur le second principe de la thermodynamique

1900 Introduction de la notion de quanta d'énergie

1918 Prix Nobel de physique

1929 Max-Planck medal

4 octobre 1947

Mort à Göttingen (Allemagne)

seur adjoint en physique théorique à I'Université de Kiel. Enfin autonome et dans une position stable, il concentre ses efforts sur l'étude de la thermodynamique et ses liens avec l'électricité et la chimie, et publie quelques articles qui font date. Il épouse en mars 1887 Marie Merck [1861-1909], une jeune fille qu'il fréquente depuis plusieurs années, et s'installe à Grunewald, dans la banlieue de Berlin. Ils auront quatre enfants : Karl, qui disparaît à Verdun en 1916 ; 
Erwin, qui sera son conseiller pendant l'entre-deux-guerres; et deux filles jumelles Margaret et Emma. Le couple reste uni jusqu'au décès de Marie, probablement de la tuberculose, en 1909. Deux ans plus tard, Planck épouse Marga von Hößlin [1882-1948], la nièce de Marie, qui lui donnera un fils, Hermann.

\section{Les travaux de recherche}

En octobre 1887, la disparition de Kirchhoff laisse vacante la chaire de physique théorique de l'Université FriedrichWilhelms de Berlin. Boltzmann [18441906] et Hertz [1857-1894] sont pressentis pour lui succéder, mais ils se désistent. Sur la recommandation chaleureuse de Helmholtz, Planck décroche le poste en 1888. C'est une position prestigieuse, et une proposition fabuleuse pour un jeune professeur. Après une sorte de période d'essai où il n'est que professeur extraordinaire, il est finalement confirmé au poste en 1892 ; il le conservera jusqu'à sa retraite en 1927, avant de passer la main à Schrödinger [1887-1961].

Planck atteint sa pleine maturité scientifique dans les années 1890. Les cours qu'ils donnent lui bâtissent une solide réputation de professeur qui traverse les frontières. Sous son impulsion, les sociétés physiques d'Allemagne se réunissent et forment la Deutsche Physikalische Gesellschaft en 1898. Sur le plan scientifique, il reste hanté par le second principe de la thermodynamique, et s'intéresse à la loi de rayonnement thermique du corps noir - une des deux grandes énigmes de la physique du $19^{\mathrm{e}}$ siècle, selon Lord Kelvin [1824-1907]. En effet, les calculs de l'énergie totale émise par un corps noir mènent au résultat forcément erroné qu'il émet une quantité infinie d'énergie. Cette divergence numérique apparaît lors de l'intégration du spectre aux courtes longueurs d'ondes, d'où son nom de catastrophe ultraviolette. Max Planck en conclut que le modèle classique du rayonnement thermique, basé sur I'hypothèse d'un spectre continu, est faux... C'est pour lui, ainsi qu'il l'écrira, un acte désespéré qui ébranle sa croyance profonde en I'universalité du principe d'entropie, mais il s'y résout car il le mène à une interprétation théorique valide. Nous sommes alors en octobre 1900. Planck introduit la notion de quanta d'énergie : il fait l'hypothèse que l'énergie n'est pas émise de manière continue, mais par paquets dont la valeur dépend de la longueur d'onde. C'est une remise en cause fondamentale du dogme de continuité des lois de la physique.

En combinant les formules de Wien [1864-1928] et de Rayleigh [1842-1919], il parvient alors à établir la loi, qui porte aujourd'hui son nom, de répartition spectrale du rayonnement thermique du corps noir. Deux mois plus tard, poursuivant sur sa lancée, il définit quatre grandeurs physiques fondamentales (ou échelles de Planck) basées sur les constantes universelles, dont celle qu'il vient de découvrir, $h$. Sans vraiment maîtriser le sens physique de la notion de quanta, dont il sent pourtant qu'elle doit jouer un rôle fondamental en physique, il présente ses résultats le 14 décembre 1900 devant la toute jeune Deutsche Physikalische Gesellschaft à Berlin. C'est la naissance de la théorie des quanta.

Mais Planck, alors âgé de 42 ans, ne parvient pas à assumer sa propre hypothèse rendant la physique discontinue et s'efface de la scène. La théorie quantique s'épanouira sans lui, avec d'autres : Einstein et les quanta de lumière, Poincaré et la nécessité mathématique des quanta induite par la loi du rayonnement de Planck, Bohr et la théorie de l'atome... Les quanta de Planck s'avèrent extraordinairement efficaces pour interpréter les observations expérimentales, et marquent un tournant dans I'histoire de la physique. Sa notoriété est immense et son travail séminal universellement reconnu. Avec Nernst [1864-1941], il organise en novembre 1911 le premier congrès Solvay à Bruxelles. Après avoir été nommé deux fois en 1907 et en 1908, il reçoit le prix Nobel de physique de 1918 «en reconnaissance des services rendus à l'avancement de la physique par sa découverte des quanta d'énergie». Notons également qu'il a été le directeur de thèse de deux Prix Nobel, von Laue [1879-1960] et Bothe [1891-1957].

\section{Osics}

\section{Plate-forme}

Modulaire

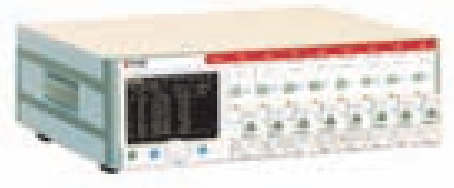

Nouveaux Modules:

- Obturateurs

- Commutateurs

- Atténuateurs haute puissance $(2 \mathrm{~W})$

- Simulateur de réflexion

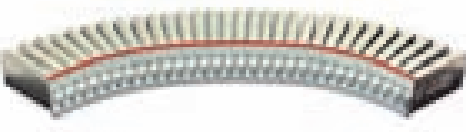

Large choix de sources:

- Lasers DFBs télécom

- Lasers accordables à cavité externe (Tunics) :

$\Rightarrow 1270-1650 \mathrm{~nm}$

$\Rightarrow 1 / 10 \mathrm{pm}$ de résolution

$\Rightarrow$ Raie $<150 \mathrm{kHz}$

$\Rightarrow$ SSE $>90 \mathrm{~dB} / 0.1 \mathrm{~nm}$

\section{Tenista}

Tel: +33 (0)1.96.48.37.19 sales-france@yenista.com

www.yenista.com 


\title{
Nouveauté
}

\section{DÉMOGRAPHIE, CLIMAT}

\section{ET ALIMENTATION MONDIALE}

\author{
Sous la direction de Henri Leridon \\ et Ghislain de Marsily
}

L'humanité trouvera-t-elle de quoi nourrir 9 milliards d'hommes en 2050, dans un contexte climatique probablement plus difficile ? L'inquiétude n'est pas nouvelle, mais la croissance démographique s'est emballée dans la seconde moitié du XX siècle, et la crainte d'une crise alimentaire mondiale est réapparue.

Certes, les progrès des technologies agricoles ont permis, au plan global, de maintenir le niveau nutritionnel moyen pendant les années de la "Révolution verte ", mais la situation restait loin d'être satisfaisante, puisque environ 850 millions de personnes étaient sous-alimentées au début du XXI siècle. Or de nouvelles inquiétudes se font jour : les surfaces cultivables ne sont plus guère extensibles, la productivité des sols atteint des niveaux qui risquent de les endommager de façon irréversible, les prix de l'énergie et des intrants indispensables à l'agriculture moderne sont à la hausse... Et les perspectives de changements climatiques ne sont pas rassurantes. De nouveaux progrès technologiques permettront-ils de dépasser, une fois encore, les contraintes naturelles et démographiques?

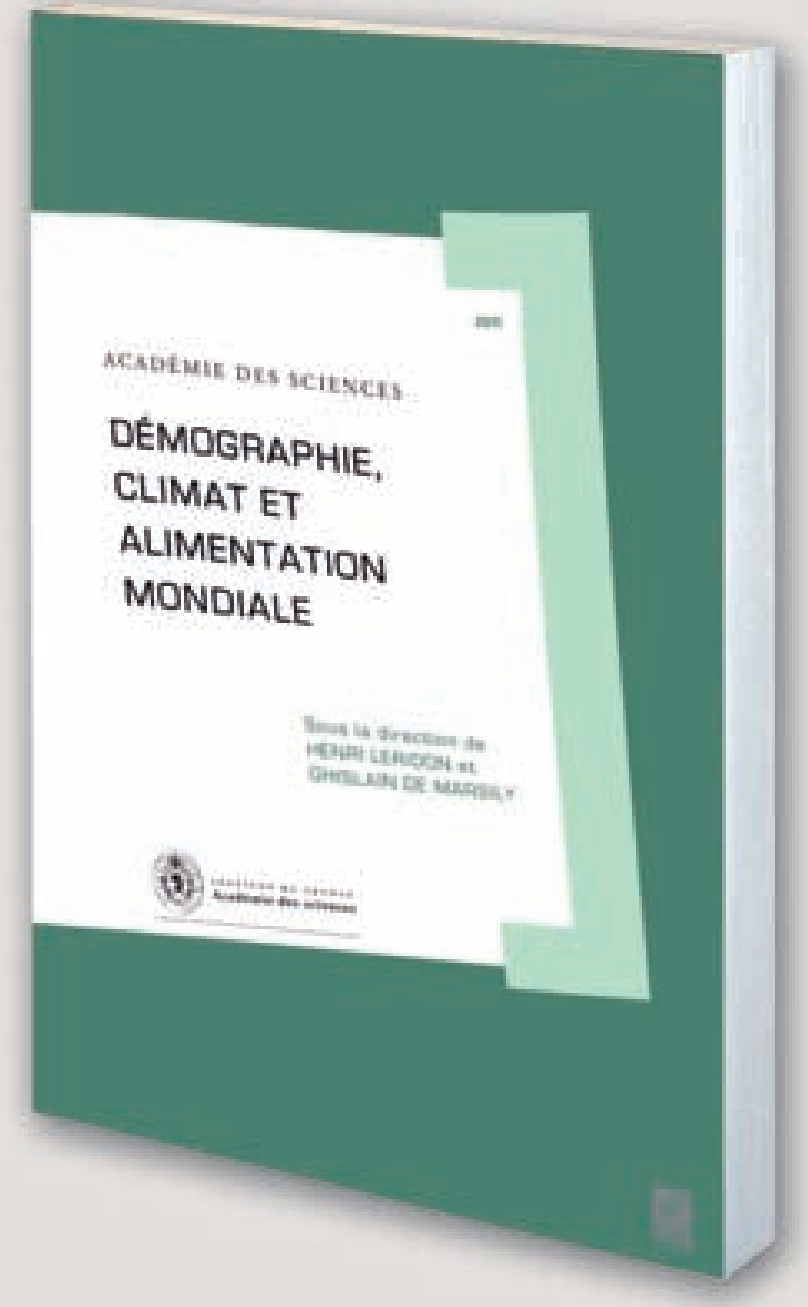

Cet ouvrage rappelle d'abord ces contraintes démographiques, climatiques et environnementales qui pèseront sur la disponibilité de ressources à l'horizon 2050, et traite ensuite des facteurs d'évolution de la demande alimentaire, des moyens techniques et économiques d'y faire face, et enfin des conditions d'un équilibre possible. Une synthèse est proposée en début de volume.

Des recommandations y sont faites, destinées aux pouvoirs publics français pour les éclairer sur leur politique nationale et internationale, mais aussi à la société civile, qui est concernée car les problèmes ne sont pas seulement scientifiques ou technologiques : ils mettent aussi en jeu les comportements individuels des producteurs et des consommateurs. Trois domaines où il faut agir sans tarder sont mis en valeur : la nutrition et les habitudes alimentaires, puis la démographie, et enfin l'économie mondiale et le commerce international. Les recommandations portent ensuite sur la production agricole et les questions d'environnement, puis sur la prévention, l'anticipation et la gestion des situations de crise, hélas quasi inévitables.

- Collection Académie des sciences - Rapport sur la science et la technologie $n^{\circ} 32$

-978-2-7598-0581-5 • 394 pages (illustrations couleur)

Parution
le 10 mars 2011 


\section{Une vie au service de la science}

Planck s'investit fortement pour l'essor des sociétés savantes en Allemagne. En 1894, il est élu membre de l'Académie royale des sciences et des lettres de Berlin, dont il est nommé secrétaire perpétuel du comité de physique en 1912. En 1913, il est nommé recteur de l'Université de Berlin. En 1930, à la mort de von Harnack, Planck est nommé président de Kaiser Wilhelm Gesellschaft.

Puis l'Europe s'enflamme. Comme un capitaine fidèle au poste, tentant de son mieux de préserver les acquis scientifiques, Planck ne fuit pas I'Allemagne nazie. Mais la fin de la guerre l'éprouve durement : sa maison de Berlin est détruite par un raid aérien en février 1944, emportant des archives scientifiques inestimables. Cette même année, son fils Erwin est arrêté, accusé de tentative d'assassinat sur Hitler dans le cadre du complot du 20 juillet 1944. Il est torturé par la Gestapo, et meurt en février 1945. Après la tempête, Planck, âgé de 87 ans, est ramené à Göttingen par les alliés. II se met sans hésiter à la tâche et aide à la reconstruction des organismes scientifiques de l'Allemagne. Il accepte à nouveau la charge de Président de la Kaiser Wilhelm Gesellschaft. Sous sa houlette, la vénérable institution, qui a survécu péniblement aux remous politiques du second conflit mondial, parvient à renaître. En reconnaissance, elle devient en 1948 la Max Planck Gesellschaft, et est aujourd'hui, l'une des grandes institutions de la recherche fondamentale en Allemagne.

\section{Les honneurs}

En 1921, Planck est lauréat de la médaille Liebig décernée par la Société des chimistes allemands, puis en 1927 de la médaille Franklin pour sa découverte des quanta d'énergie. Il est élu membre étranger de la Royal Society de Londres en 1926, dont il reçoit la prestigieuse médaille Copley en 1928. II reçoit en 1927 la Médaille Lorentz, prix décerné par I'Académie royale des arts et des sciences néerlandaise. Honneur considérable, la médaille Max Planck de physique est créée en 1929, et il en est avec Einstein [1879-1955] le premier récipiendaire.

Depuis son jeune âge, Planck montre un goût marqué pour la musique. II maîtrise le piano, et donne parfois des concerts chez lui sur un harmonium qu'il s'est fait construire. Il compose également quelques pièces musicales. Ce n'est probablement pas un hasard si ce scientifique amoureux des sons a su, si élégamment, lever un coin du voile sur l'harmonie secrète de la nature.

Révéré par ses collègues, auréolé d'une immense gloire, Max Planck s'éteint le 4 octobre 1947 à Göttingen.

\section{Riad HAIDAR \\ Onera \\ haidar@onera.fr}

\section{Références}

[1] JL Heilbron, Planck, la révolution quantique, Les génies de la science, 2006, 27, p. $32-120$.

[2] Nobel lectures, http://nobelprize.org

\section{Caméra Intensifiée Obturable C10880-03}

\section{Contrôle de combustion sur moteur à explosion}

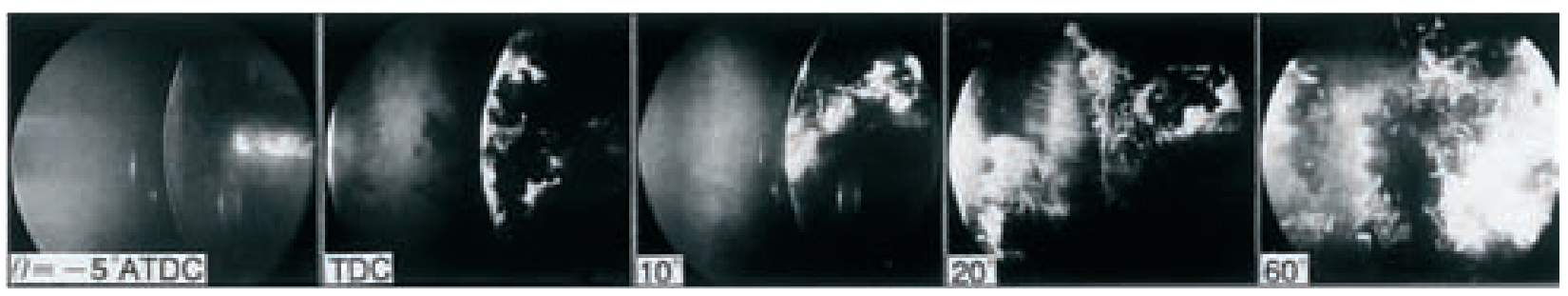

\section{Spécifications :}

- Temps d'obturation 10ns

- Fréquence de répétition : $200 \mathrm{kHz}$

- Multi expositions

- Image booster et générateur d'impulsions intégrés

\section{www.hamamatsu.fr}

infos@hamamatsu.fr - Tél. +33169537100

\section{Applications :}

- Etude de propagation de flamme dans les cylindres

- Observation des jets de carburant des systèmes d'injection 